D.O.I.: $10.3895 / \mathrm{S} 1808-04482009000300008$

\title{
CONTRIBUIÇÃO DA METODOLOGIA MULTICRITÉRIO DE APOIO À DECISÃO NO MÉTODO DO FLUXO DE CAIXA DESCONTADO USADO PARA AVALIAR EMPRESAS DE PEQUENO PORTE
}

\section{CONTRIBUTION METHODOLOGY MULTICRITERIA DECISION AID IN THE METHOD OF DISCOUNTED CASH FLOW USED TO EVALUATE SMALL BUSINESSES}

\author{
Marcus Vinicius Andrade de Lima ${ }^{1}$; Ana Lúcia Miranda Lopes ${ }^{2}$; Ademar Dutra ${ }^{3}$ \\ ${ }^{1}$ Universidade do Sul de Santa Catarina - UNISUL - Florianópolis - Brasil \\ marcus.lima@unisul.br \\ ${ }^{2}$ Universidade Federal de Minas Gerais - UFMG - Belo Horizonte - Brasil \\ analopes@face.ufmg.br \\ ${ }^{3}$ Universidade do Sul de Santa Catarina - UNISUL - Florianópolis - Brasil \\ ademar.dutra@unisul.br
}

\begin{abstract}
Resumo
Na busca de uma avaliação em negócios mais ampla, se fez necessário incorporar no fluxo de caixa descontado variáveis de natureza qualitativa e subjetiva. Portanto, o objetivo desse artigo é contribuir na aplicação do método do fluxo de caixa descontado, utilizando a metodologia multicritério de apoio à decisão. Para atingir esse objetivo foi realizado um estudo descritivo de natureza exploratória aplicado a um multicaso. A intervenção foi em empresas de pequeno porte do ramo químico, farmacêutico e de turismo. Como resultado, o avaliador ajustou o preço do negócio levando em conta a resultante da mixagem entre as duas metodologias.
\end{abstract}

Palavras-chave: metodologia multicritério; fluxo de caixa descontado; balanço inventariado; empresas de pequeno porte.

\section{Introdução}

O preço de uma empresa, referência fundamental em qualquer negociação, nunca é arbitrário. Ao contrário, sua determinação exige a combinação da análise estratégica do negócio com modernas teorias de finanças. Portanto, o valor de uma empresa é o quanto, potenciais adquirentes, estariam dispostos a pagarem pela compra do patrimônio líquido de uma empresa (SANTOS, 2005). Neste caso, o valor de uma empresa não é uma posição unilateral do proprietário, mas sim das partes interessadas. É importante salientar que os princípios de avaliação contábil não foram feitos para medir o valor de venda de uma empresa. Pelo contrário, o princípio do custo com base no valor dos ativos está voltado à mensuração do lucro e não à medida do seu valor de venda 
(IUDÍCIBUS, 1997). Se o valor da empresa depende dos seus lucros futuros, seria impossível à contabilidade registrá-lo, dado o alto grau de incerteza provocado pelo fato desse valor depender dos lucros projetados, taxa de juros da economia e da subjetiva taxa de risco atribuída ao empreendimento.

Ao decidir, entende-se que certa dose de subjetividade esteja interagindo com os aspectos objetivos durante o processo decisório. Portanto, ao considerar esse contexto, julga-se importante à contribuição de uma metodologia que permita incorporar esses aspectos ao se tomar decisões e que eles possam ser explicitados e quantificados.

A Metodologia Multicritério de Apoio à Decisão (MCDA) é um dos métodos por excelência no qual tal simbiose pode se materializar. Ela consiste em um conjunto de métodos e técnicas que auxiliam ou apóiam pessoas e organizações a tomarem decisões, sob a influência de uma multiplicidade de variáveis.

Métodos de avaliação de empresas de grande porte podem ser encontrados nas obras de Cupertino et al (2006); Sousa, Bastos e Martelanc (2003); Copeland, Kooller e Murrin (2000); Damodaram (1999) e Pratt (1989) nas quais são largamente discutidas questões importantes e complexas de avaliação de empresas de capital aberto e fechado. Entretanto, raramente encontramse métodos específicos para avaliação de Empresas de Pequeno Porte (EPPs) na literatura brasileira. Não obstante a fragilidade dos números apresentados, quando eles existem, esses demonstrativos financeiros elaborados dentro dos princípios contábeis e éticos podem não expressar a realidade dos negócios.

A metodologia denominada de "balanço perguntado" ou "balanço inventariado" (KASSAI e KASSAI, 2004 e CAIXA ECONÔMICA FEDERAL, 2000), pode ser uma alternativa para as EPPs. Trata-se de uma metodologia para levantamento de informações por meio de um questionário previamente elaborado que permite diagnosticar a sua situação econômica e financeira, condição indispensável à avaliação de empresas.

Portanto, o artigo tem o objetivo de utilizar a MCDA como uma contribuição para o método do Fluxo de Caixa descontado (FCD) utilizado na avaliação de EPPs. Dessa forma, pretende-se identificar o valor de negociação (preço) mais "justo" em decorrência de poder incorporar no processo de avaliação as variáveis qualitativas e subjetivas as quais são inerentes ao avaliador. Para que este objetivo se materializasse, realizou-se um estudo descritivo de natureza exploratória aplicado a um multicaso. As informações necessárias para a pesquisa forma obtidas em EPPs do ramo químico, farmacêutico e de turismo.

Nas demais seções do artigo serão apresentadas às bases teóricas do estudo, os procedimentos metodológicos empregados na avaliação de empresas de pequeno porte, uma aplicação dos métodos utilizados, as limitações dos métodos e as considerações finais dessa 
investigação.

\section{Pressupostos Teóricos}

O Método do Fluxo de Caixa Descontado está fundamentado na idéia de que o valor de uma empresa está diretamente relacionado aos montantes e às épocas em que os fluxos de caixa operacionais estarão disponíveis para os detentores de capital. Portanto, o valor de uma empresa é medido pelo montante de recursos financeiros que será gerado no futuro pelo negócio, o qual é trazido ao seu valor presente para refletir o tempo e o risco associado ao investimento (MARTELANC, PASIN, CAVALCANTE; 2005). Copeland, Koller e Murrin (2000) descrevem os seguintes passos para a avaliação de empresas de acordo com o esse método: (1) analisar o desempenho histórico; (2) projetar o desempenho futuro e o correspondente fluxo de caixa; (3) estimar o custo de capital; (4) estimar o valor da perpetuidade; (5) calcular e interpretar os resultados. A primeira etapa para avaliar uma empresa é analisar seu desempenho histórico. Uma noção sólida do desempenho da empresa no passado oferece uma perspectiva essencial para o desenvolvimento de projeções criteriosas sobre o desempenho futuro. Nesta fase é importante fazer um ajuste na demonstração de resultados, destacando o fluxo de caixa descontado da empresa e do proprietário. A segunda etapa é projetar o desempenho futuro da empresa e o seu correspondente fluxo de caixa. Apesar de não se ter regra específica, pode-se considerar algumas etapas básicas como: (1) estimar o potencial de crescimento da empresa e a capacidade de obter retornos acima do seu custo de capital; (2) desenvolver cenários de desempenho para a empresa; (3) projetar rubricas individuais da demonstração de resultados e do balanço patrimonial com base nos cenários desenvolvidos e (4) utilizar o bom senso nas projeções como um todo, principalmente nas principais estratégias adotadas. A terceira etapa é estimar o custo de capital (TOMAZONI e MENEZES, 2002) ou custo médio ponderado de capital (WACC - weighted average cost of capital). Representa o custo de oportunidade da empresa obtido pela ponderação dos custos de capitais de terceiros $(K i)$ e próprio $(K e)$ com as respectivas participações no total do capital (passivo mais patrimônio líquido). Determina-se o $K i$ através da ponderação do custo das diversas dívidas onerosas, descontado o beneficio fiscal. Para o cálculo do Ke foi adotado como alternativa o CAPM (capital asset price model), desenvolvido por Sharpe e Markowitz, de tal forma que este método possibilita estimar a taxa de retorno que os proprietários esperam obter sobre o capital investido em função do risco do negócio. É dado pela seguinte fórmula ajustada (DAMODARAN, 1999): $K_{e}=R_{f}+\beta \cdot\left(R_{m}-\right.$ $\left.R_{f}\right)+\alpha_{\text {pais }}$; onde $K_{e}$ é o retorno mínimo exigido pelos proprietários; $R_{f}$ é a taxa livre de risco; $\beta$ é o coeficiente beta, que representa o risco sistemático; $R_{m}$ é a taxa de retorno de mercado; $\alpha_{\text {pais }}$ é um prêmio adicional que reflete o risco de se investir em países emergentes (diferença entre a taxa de 
um título do tesouro dos EUA e um título da dívida emitido pelo governo do país em questão). Portanto, o WACC é a taxa utilizada para descontar os fluxos de caixa futuros a valor presente para todos os investidores. A quarta etapa é estimar o valor da perpetuidade que é o valor dos fluxos de uma empresa além do período de projeção explicita. O cálculo do valor da perpetuidade elimina a necessidade de se prever em detalhes os fluxos de caixa de uma empresa por um período excessivamente longo. Pode-se calcular com base na média dos últimos três anos (ASSAF NETO, 2006) e incrementado pela expectativa de crescimento (g). Finalmente, a quarta etapa do processo de avaliação envolve calcular e testar o valor da empresa e depois interpretar os resultados em relação ao contexto da decisão.

A operacionalização dos fundamentos da MCDA é realizada por meio de três fases básicas: (1) estruturação do contexto decisório; (2) a construção de um modelo de avaliação das alternativas (empresas) e a (3) formulação de recomendações para possíveis ajustes naqueles pontos considerados fundamentais para o decisor (avaliador). Na fase 1, estruturação do contexto decisório deve ser identificada os atores envolvidos na tomada de decisão e escolhidos os decisores que participarão ativamente na construção do modelo. A seguir, é necessário identificar o tipo de alternativa (empresa) que será avaliada e a problemática que ela está inserida. Várias são as técnicas que podem ser usadas a fim de extrair do decisor aqueles pontos que ele considera fundamental para a tomada de decisão. Uma das técnicas mais usadas é o mapa cognitivo ou de relação meios e fins. Esse mapa serve para auxiliar a representar o problema do decisor cujo objetivo é identificar aqueles pontos que ele considera fundamental na composição do mesmo. Uma vez identificados os pontos de vistas fundamentais (PVF), é possível representá-lo na forma de uma estrutura arborescente, onde é possível aumentar o grau de compreensão do decisor. Desta forma constrói-se uma estrutura de valor ou uma árvore de pontos de vistas. Outros autores utilizam nomenclaturas diversas para descrever tal estrutura arborescente (ver BELTON, 1990; KEENEY, 1996; GOODWIN e WRIGHT, 1991). Uma vez construída a estrutura de valor é necessário um critério que permita mensurar o desempenho de cada alternativa avaliada em cada ponto de vista considerado. Cada critério é decomposto em diversos níveis $(\mathrm{Nn})$ chamados de descritores os quais fornecem um melhor entendimento daquilo que representa a preocupação do decisor ao mensurar uma dimensão do contexto decisório. Uma vez construídos os descritores, passa-se para a fase 2, construção do modelo de avaliação das alternativas (empresas). Para isso é necessário quantificar o desempenho das alternativas (empresas) em relação a cada critério identificado pelo avaliador e decomposto no diversos níveis dos descritores. O instrumento que pode auxiliar os decisores a expressar, de forma numérica, suas preferências será a função de valor. Isso é necessário tanto para avaliar as alternativas, quanto melhorar o entendimento sobre o problema dos decisores. Uma vez quantificados estes critérios, é possível agregar estas informações locais de modo a obter uma 
avaliação global. Para que seja possível esta agregação é necessário um conjunto de parâmetros associados aos critérios os quais são chamados de taxas de compensação. Essas taxas são parâmetros que os decisores julgam adequados para agregar, de forma compensatória, desempenhos locais em um desempenho global (BOUYSOU, 1989; ROY, 1996). Elaborada a avaliação local das alternativas, em todos os pontos de vistas, é importante, agora utilizar esta informação para comparar alternativas potenciais e determinar seus pontos fortes e fracos. Para uma melhor visualização do desempenho de cada alternativa potencial, traça-se o seu perfil de impacto (BELTON, 1990). O perfil de impacto é especialmente útil, para, além de avaliar as alternativas, possibilitar a geração de oportunidades para aperfeiçoá-las, favorecendo, com isso, o aumento de conhecimentos dos decisores sobre o seu problema. Essas avaliações locais podem ser agregadas agora em uma única avaliação global. Isso pode ser feito através da fórmula de agregação aditiva (KEENEY, 1996). A partir do conhecimento dos pontos de vistas fundamentais onde o desempenho da alternativa mostrou-se inadequado, torna-se possível concentrar esforços para correção ou proposição de novas alternativas para melhoria deste desempenho. Esta é a função da fase 3, elaboração das recomendações.

O "balanço perguntado ou balanço inventariado" (KASSAI e KASSAI, 2004 e CAIXA ECONÔMICA FEDERAL, 2000), trata-se de uma metodologia para o levantamento de informações por meio de um questionário previamente elaborado e que permite diagnosticar a situação econômica e financeira de uma determinada empresa. Essa técnica é uma prática existente no mercado e surgiu, provavelmente, da constatação de que os relatórios contábeis apresentados pelas EPPs pudessem não espelhar a sua realidade. Algumas instituições de créditos para EPPs e órgãos ligados ao Serviço Brasileiro de Apoio às Micro e Pequenas Empresas (SEBRAE), destacam os seguintes itens sob a forma de balanço perguntado ou inventariado: dados cadastrais; dados econômicos e financeiros; quadro das dívidas da empresa ou proprietário(s); perspectivas do negócio a médio e a longo prazo; entre outros. Por último, a eficácia desses questionários fica em torno da veracidade dos dados informados e é fundamental proceder a uma checagem de consistência, onde a própria estrutura contábil permite verificar se os números são ou não coerentes. Por exemplo, o total do ativo deve ter uma relação com a estrutura de capital; o capital circulante líquido deve ser coerente com os prazos informados; a margem de lucro deve ser compatível com o histórico das retiradas e pró-labore; as taxas de retornos apurados têm como referência as taxas praticadas no mercado financeiro e de capitais; entre outros.

Foi com esta base teórica que a investigação da pesquisa pôde ser realizada. Na próxima seção, serão descritos os procedimentos metodológicos que permitiram atingir os objetivos do artigo operacionalmente. 


\section{Procedimentos Metodológicos da Pesquisa}

Tendo em vista o problema de avaliação das EPPs, onde a fragilidade das informações econômico-financeiras parece ser predominante e que os aspectos qualitativos e subjetivos existem em abundância, o método utilizado nesta investigação foi, predominantemente, o qualitativo. Foi realizado um estudo descritivo de natureza exploratória para que o objetivo desta investigação fosse atingido. Utilizou-se de entrevistas semi-estruturadas como instrumento de coleta de dados. Durante as entrevistas houve muita interação e flexibilidade com os proprietários e dirigentes, possibilitando extrair informações necessárias, dentro de cada contexto. Coletou-se um número significativo de informações, cuja finalidade era identificar o valor dessas empresas como negócio.

Como foram estudadas mais de uma empresa, a investigação caracterizou-se por um estudo multicaso. Fez-se a análise de três EPPs, uma do setor químico, a outra do setor farmacêutico e a última do setor de turismo. Para as escolhas dessas empresas, primeiramente definiu-se aquelas que tivessem uma receita bruta anual de até $\mathrm{R} \$ 2.400 .000,00$ e, em segundo lugar, escolheu-se aquelas que pudessem fornecer informações completas e confiáveis.

Tendo confirmada a possibilidade de realização da pesquisa, foram feito questionários que destacam as informações mais relevantes sobre um balanço patrimonial inventariado ou perguntado, a saber: dados cadastrais; econômicos e financeiros; quadro das dívidas da empresa e perspectivas do negócio a médio e a longo prazo. Sempre se procedia a uma checagem de consistência na busca da veracidade das informações obtidas. Após a tabulação dos dados chegou-se a um balanço patrimonial (BPi) e uma demonstração de resultados (DRi) perguntado ou inventariado.

De posse desses dois relatórios financeiros (BPi e DRi), partiu-se para a projeção dos fluxos de caixas para um período de 10 anos e no cálculo do valor presente foi considerada também a perpetuidade das empresas. Inicialmente projetou-se o lucro operacional genuíno (ASSAF NETO, 2006) das empresas e a partir dele mediante uma série de ajustes, estimou-se o fluxo de caixa. Esse lucro é como se a empresa não possuísse qualquer dívida, exceto talvez empréstimos de curto prazo e de pequeno valor, utilizados para cobrir desajustes de caixa provenientes da sazonalidade do negócio. O primeiro ajuste foi descontar deste lucro os impostos sobre o lucro real, os quais as empresas teriam se não possuíssem quaisquer dívidas estruturais. Outros ajustes envolveram a soma da depreciação, por não apresentarem uma saída de caixa efetiva, que é compensada pela inclusão, como saída de caixa, dos investimentos necessários para que não seja alterado o valor das empresas. Uma eventual defasagem temporal devida ao efeito dos prazos de recebimento, de pagamento e dos estoques foi ignorada, porque traz pequenos efeitos sobre o valor presente das empresas.

Para o cálculo da taxa de desconto foi considerada a média ponderada do custo de capital de terceiros e próprio. A ponderação levou-se em conta o volume de recursos contidos no passivo 
oneroso e no patrimônio líquido da estrutura de capital dessas empresas. O custo de capital de terceiros foi obtido através das taxas vigente praticadas pelos bancos com linha de crédito específica para o fomento de EPPs. Na ausência de um padrão brasileiro para o cálculo do custo do capital próprio, utilizou-se como benchmarking uma grande empresa brasileira (ASSAF NETTO, 2006), atualizando o risco país (C-Bond menos T-Bond) em menos dois pontos percentuais, e acrescentando também a inflação prevista pelo Banco Central do Brasil (BACEN), conforme Relatório de Inflação de Março de 2007. Usando este mesmo relatório estimou-se a taxa de crescimento constante $(g)$ para o cálculo da perpetuidade do fluxo de caixa das empresas, tomando como base o PIB previsto pelo governo brasileiro.

Finalmente, utilizou-se a MCDA para que contribuísse com o método do FCD, na inclusão de variáveis qualitativas e subjetivas consideradas relevantes pelo avaliador, no momento da sua decisão final. A tarefa mais árdua foi à extração dessas variáveis as quais não estavam incluídas na projeção do fluxo de caixa, mas que o avaliador levava em conta no momento da sua avaliação. Várias foram às entrevistas até que se conseguiu elaborar, de forma didática, uma estrutura de valor que expressasse esses pontos de vistas considerados fundamentais para o avaliador.

Definidos esses conjuntos de pontos de vistas fundamentais, passou-se a descrevê-lo com o objetivo de fornecer um melhor entendimento daquilo que representa a preocupação do avaliador no contexto decisório. Após elaborar esses descritores e com o auxílio do software Macbeth (BANA e COSTA e VANSNICK, 1995) foram construídas as funções de valor, os quais são instrumentos para auxiliarem os avaliadores a expressar, de forma numérica, as suas preferências.

Construídas as funções de valor, a etapa seguinte foi agregar todos os pontos de vistas fundamentais da estrutura de valor. Para tal, foi necessário determinar as taxas de compensação. Essas taxas foram necessárias para agregar, de forma compensatória, as informações locais de modo a obter uma avaliação global, permitindo, com isto, comparar melhor as alternativas disponíveis. O software Macbeth (BANA e COSTA e VANSNICK, 1995) cumpriu essa tarefa.

Para uma melhor visualização do desempenho das empresas, em cada ponto de vista, traçouse um perfil de impacto (BELTON, 1990). Este gráfico, além de permitir um diagnóstico mais acurado e completo, desses desempenhos, foi especialmente útil para possibilitar a geração de oportunidades de aperfeiçoamentos. Construído o perfil de impacto foi possível proceder à avaliação global das empresas. Isso foi feito utilizando a fórmula de agregação aditiva (KEENEY e RAIFFA, 1993).

E, por último, apurado o valor global das empresas pode-se enquadrar esses valores na escala de preferência qualitativa, determinada pelo avaliador, ocasionando um ágio ou deságio no valor presente das empresas, provenientes do método do fluxo de caixa descontado. Com estes valores finais o avaliador teve condições para iniciar as rodadas de negociações. 


\section{Aplicação dos Métodos para Avaliação de Empresas de Pequeno Porte (EPPs)}

Para ilustrar as metodologias propostas, foram utilizados os dados da empresa Alfatecnoquímica de forma analítica, e as duas outras de forma sintética para análise global dos resultados. A empresa ilustrada fabrica equipamentos para análise de água e efluentes industriais. Localizada em Santa Catarina, conta com 10 colaboradores distribuídos entre as atividades de produção e administração. O proprietário domina o processo de produção e comercialização, sempre que possível utiliza parcerias e terceirização. Não foi permitido divulgar os dados financeiros da empresa a fim de preservar as estratégias adotadas. Embora se tenha procurado manter as características da empresa, os dados apresentados não expressam a realidade do negócio. Portanto, após a tabulação dos dados, a Alfatecnoquímica apresentou o balanço perguntado ou inventariado, conforme Quadro 1.

Uma vez elaborados os demonstrativos (Quadro 1), iniciou-se a projeção do fluxo de caixa, que foram trazidos a valor presente para serem computados no cálculo do valor da empresa. Algumas premissas foram levadas em conta, por exemplo, o volume de vendas, a variação dos custos de produção, o levantamento das perspectivas de mudanças tecnológicas, o dimensionamento dos investimentos futuros, o tempo de vida útil desses investimentos, a tendência de mudanças nos tributos a que o negócio está sujeito, entre outras. A quadro 2 expressa o primeiro qüinqüênio (2007 a 2011), de um período explícito de 10 anos, a projeção do fluxo de caixa tanto na visão da empresa (fluxo de caixa livre para os detentores de capitais) quanto na perspectiva do(s) proprietário(s). A Quadro 3 que apresenta o segundo qüinqüênio que vai do ano 2012 a 2016, completando, assim, o período explícito de 10 anos. Da mesma forma o fluxo de caixa projetado contempla as duas informações cruciais para avaliação: Fluxo de caixa da empresa e o fluxo de caixa do(s) proprietário (os). 
Quadro 1 - Balanço Patrimonial e Demonstração de Resultados Inventariado 2006

\begin{tabular}{|c|c|c|c|c|c|}
\hline \multicolumn{4}{|c|}{ Balanço Patrimonial } & \multicolumn{2}{|c|}{ Demons tração de Resultados } \\
\hline \multicolumn{2}{|l|}{ ATIVO } & \multicolumn{2}{|l|}{ PAS SIVO } & VENDAS & 1.500 .000 \\
\hline Circulante & & Circulante & & Custos Variáveis & \\
\hline caixa & 6.000 & fornecedores & 420.000 & impos tos & $(102.000)$ \\
\hline a receber & 720.000 & pes soal & 75.000 & compra mercadorias & $(900.000)$ \\
\hline \multirow{3}{*}{ estoques } & 180.000 & imp os tos & 72.000 & custo pessoal & $(75.000)$ \\
\hline & & ou tros & 37.200 & Marg em Contribuição & 423.000 \\
\hline & & & & Des pes as Fi xas & \\
\hline Imobilizado & & Exigi vel a longo pz & & despesas a dministrat ivas & $(30.000)$ \\
\hline móveis e ut en sílios & 360.000 & fin an ciamen to $\mathrm{s}$ & 312.000 & despesas financeiras & $(37.440)$ \\
\hline equipamentos & 540.000 & & & depreciação & $(10.008)$ \\
\hline \multirow[t]{3}{*}{ veiculos } & 190.200 & Patri mônio Líquido & & outras despesas & $(15.000)$ \\
\hline & & Capital social & 960.000 & Lucro Operacional & 330.552 \\
\hline & & Lucros acumulados & 120.000 & $\mathrm{IR}+\mathrm{CS}$ & $(128.915)$ \\
\hline TOTAL & 1.996 .200 & \begin{tabular}{|r|} 
TOTAL \\
\end{tabular} & 1.996 .200 & Lucro líquido & 201.637 \\
\hline
\end{tabular}

Fonte: Autoria própria (2007)

\begin{tabular}{|c|c|c|c|c|c|}
\hline Dados & 2007 & 2008 & 2009 & 2010 & 2011 \\
\hline VENDAS & 1.500 .000 & 1.500 .000 & 1.500 .000 & 1.900 .000 & 1.900 .000 \\
\hline \multicolumn{6}{|l|}{ Custos Variáveis } \\
\hline impostos & $(102.000)$ & $(102.000)$ & $(102.000)$ & $(129.200)$ & $(129.200)$ \\
\hline compra mercadorias & $(900.000)$ & $(900.000)$ & $(900.000)$ & $(1.140 .000)$ & $(1.140 .000)$ \\
\hline despesas pessoal & $(75.000)$ & $(75.000)$ & $(75.000)$ & $(95.000)$ & $(95.000)$ \\
\hline Margem Contribuição & 423.000 & 423.000 & 423.000 & 535.800 & $\mathbf{5 3 5 . 8 0 0}$ \\
\hline \multicolumn{6}{|l|}{ Despesas Fixas } \\
\hline despes as administrativas & $(30.000)$ & $(30.000)$ & $(30.000)$ & $(38.000)$ & $(38.000)$ \\
\hline depreciação & $(10.008)$ & $(10.008)$ & $(10.008)$ & $(10.008)$ & $(10.008)$ \\
\hline outras despesas & $(15.000)$ & $(15.000)$ & $(15.000)$ & $(19.000)$ & $(19.000)$ \\
\hline Lucro operacional & 367.992 & 367.992 & 367.992 & 468.792 & 468.792 \\
\hline $\mathrm{IR}+\mathrm{CS}$ & $(143.517)$ & $(143.517)$ & $(143.517)$ & $(182.829)$ & $(182.829)$ \\
\hline Lucro líquido operacional & 224.475 & 224.475 & 224.475 & 285.963 & 285.963 \\
\hline depreciação & 10.008 & 10.008 & 10.008 & 10.008 & 10.008 \\
\hline FC da Empres a & 234.483 & 234.483 & 234.483 & 295.971 & 295.971 \\
\hline \multicolumn{6}{|l|}{ Financiamentos } \\
\hline Amortização & $(62.400)$ & $(62.400)$ & $(62.400)$ & $(62.400)$ & $(62.400)$ \\
\hline despesas financeiras & $(37.440)$ & $(29.952)$ & $(22.464)$ & $(14.976)$ & $(7.488)$ \\
\hline Benficio fiscal & 14.602 & 11.681 & 8.761 & 5.841 & 2.920 \\
\hline FC dos Proprietários & 149.245 & 153.812 & 158.380 & 224.436 & 229.003 \\
\hline
\end{tabular}

Uma vez projetado o fluxo de caixa da Alfatecnoquímica o próximo passo foi à identificação do seu custo total de capital. Obteve-se a partir da soma do patrimônio líquido (\$1.080.000) mais o seu passivo oneroso (\$312.000). A alíquota do imposto de renda (IR) estava na faixa de $35 \%$. As linhas de créditos para fomento de EPPs estavam na faixa de $21 \%$ a.a. e o custo de capital de terceiros (Ki) ficou em torno de 14\% a.a (com o beneficio fiscal da dívida). A taxa mínima de atratividade exigida pelos proprietários $(\mathrm{Ke})$ foi de $18,1 \%$ a.a, composta da seguinte forma: 16,1\% a.a (benchmarking; ASSAF NETTO, 2006), menos 2\% (taxa de risco país ajustada, DAMODARAN, 1999) e mais 4\% (estimativa de inflação, BACEN 2007). Portanto, através do cálculo da média ponderada do custo de capital (WACC) obteve-se a taxa de 17,1\% a.a. como custo 
total de capital da empresa.

De posse da taxa que representa do custo total da empresa (WACC), pode-se fazer uso do método do FCD para calcular o valor da Alfatecnoquímica, dividido em duas partes: fluxo de caixa projetado para o período explícito de10 anos, e o fluxo de caixa da perpetuidade, conforme ilustra a Quadro 4.

Quadro 3 - Fluxo de Caixa Projetado 2012 a 2016

\begin{tabular}{|c|c|c|c|c|c|}
\hline Dados & 2012 & 2013 & 2014 & 2015 & 2016 \\
\hline VENDAS & 1.900 .000 & 2.100 .000 & 2.100 .000 & 2.100 .000 & 2.100 .000 \\
\hline \multicolumn{6}{|l|}{ Custos Variáveis } \\
\hline impostos & $(129.200)$ & $(142.800)$ & $(142.800)$ & $(142.800)$ & $(142.800)$ \\
\hline compra mercadorias & $(1.140 .000)$ & $(1.260 .000)$ & $(1.260 .000)$ & $(1.260 .000)$ & $(1.260 .000)$ \\
\hline despesas pessoal & $(95.000)$ & $(105.000)$ & $(105.000)$ & $(105.000)$ & $(105.000)$ \\
\hline Margem Contribuição & 535.800 & 592.200 & 592.200 & 592.200 & 592.200 \\
\hline \multicolumn{6}{|l|}{ Despes as Fixas } \\
\hline despes as adminis trativas & $(38.000)$ & $(42.000)$ & $(42.000)$ & $(42.000)$ & $(42.000)$ \\
\hline depreciação & $(10.008)$ & $(10.008)$ & $(10.008)$ & $(10.008)$ & $(10.008)$ \\
\hline outras despesas & $(19.000)$ & $(21.000)$ & $(21.000)$ & $(21.000)$ & $(21.000)$ \\
\hline Lucro operacional & 468.792 & 519.192 & 519.192 & 519.192 & 519.192 \\
\hline $\mathrm{IR}+\mathrm{CS}$ & $(182.829)$ & $(202.485)$ & $(202.485)$ & $(202.485)$ & $(202.485)$ \\
\hline Lucro líquido operacional & 285.963 & 316.707 & 316.707 & 316.707 & 316.707 \\
\hline depreciação & 10.008 & 10.008 & 10.008 & 10.008 & 10.008 \\
\hline FC da Empres a & 295.971 & 326.715 & 326.715 & 326.715 & 326.715 \\
\hline \multicolumn{6}{|l|}{ Financiamentos } \\
\hline Amortização & 0 & 0 & 0 & 0 & 0 \\
\hline despesas financeiras & 0 & 0 & 0 & 0 & 0 \\
\hline Benficio fiscal & 0 & 0 & 0 & 0 & 0 \\
\hline FC do Proprietário & 295.971 & 326.715 & 326.715 & 326.715 & 326.715 \\
\hline
\end{tabular}

Fonte: Autoria própria (2007)

Quadro 4 - Valor da Empresa em 2006

\begin{tabular}{|l|r|r|r|r|r|}
\hline Fluxo de caixa des contado & \multicolumn{2}{|l|}{} & \multicolumn{1}{|c|}{} \\
\hline Empresa & \multicolumn{1}{|c|}{$\mathbf{2 0 0 6}$} & $\mathbf{2 0 0 7 - 0 9}$ & $\mathbf{2 0 1 0 - 1 2}$ & $\mathbf{2 0 1 3 - 1 6}$ & Perpetuidade \\
\hline W ACC & $17,10 \%$ & & & & \\
\hline Fluxo de caixa & & 234.483 & 295.971 & 326.715 & 326.715 \\
\hline Fluxo descontado (2006) & 1.272 .814 & & & & \\
\hline Perpetuidade (2006) & 463.610 & & & & \\
\hline Valor da Empres a & $\mathbf{1 . 7 3 6 . 4 2 4}$ & & & & \\
\hline
\end{tabular}

Fonte: Autoria própria (2007)

Para a construção dessa perpetuidade (Quadro 4), levou-se em conta a média dos três últimos anos do fluxo de caixa projetado e uma taxa de crescimento constante de $3 \%$ a.a. (BACEN, 2007).

Apurado o valor de referência da empresa é importante que se faça um ajuste do valor às condições exatas no momento da venda (contas a receber e a pagar, encargos trabalhistas, estoques, obsolescência do imobilizado, entre outros), em especial deduzindo o montante das dívidas financeiras. 
Com o objetivo de contribuir no aperfeiçoamento do método do fluxo de caixa descontado, foi incluído, no modelo, variáveis qualitativas e subjetivas consideradas fundamentais pelo avaliador, cuja intenção é a busca por uma decisão mais bem fundamentada. A MCDA fez esta simbiose entre as duas dimensões: os aspectos quantitativos do método do fluxo de caixa descontado e os interesses e valores do avaliador. Ao final foi possível que estas duas dimensões pudessem ser agregadas de modo a obter uma avaliação global da empresa como negócio.

O primeiro passo foi extrair do avaliador pontos de vistas considerados essenciais e desejáveis para serem levados em conta no processo de avaliação das empresas. Depois de exaustivas entrevistas pôde-se obter tais considerações, e a título de ilustração, conforme Quadro 5, construiu-se uma estrutura de valor (KEENEY, 1996) que serão incluídos no modelo multicritério.

Quadro 5 - Estrutura de valor

\begin{tabular}{l} 
Avaliação qualitativa \\
Contexto \\
PVF1-Produto interno bruto \\
\hline PVF2-Inflação \\
PVF3-Juros reais \\
Negócio \\
PVF4-Setor econômico \\
\hline PVF5-Clientes \\
\hline PVF6-Concorrentes \\
\hline PVF7-Fornecedores \\
\hline Capacidade dos dirigentes \\
PVF8-Visão de negócio \\
\hline PVF9-Experiência \\
\hline PVF10-Honestidade \\
Ris cos potenciais \\
PVF11-Garantias \\
\hline Fonte: Autoria própria (2007)
\end{tabular}

A segunda etapa foi construída uma base de comparação (critérios) que permita mensurar o desempenho das alternativas (empresas) em cada ponto de vista fundamental (PVF) definido pelo avaliador (Quadro 5). Para construir esses critérios, duas ferramentas são necessárias: um descritor (D) e a sua função de valor (FV). O descritor é composto de níveis (Nn) no sentido de melhor para pior. Dois níveis de referência $(\mathrm{P})$ foram definidos: Bom $(\mathrm{B})$ e Neutro $(\mathrm{N})$. Essas referências foram utilizadas para reconhecer o desempenho de cada alternativa com nível de excelência (acima do Bom), competitivo (entre o nível Bom e Neutro) e comprometedor (abaixo do Neutro). Na seqüência, foi a construção da função de valor, que constituiu uma forma numérica de expressar a intensidade das preferências do avaliador em cada nível do descritor. A literatura apresenta vários métodos para a construção de valor. Neste trabalho foi usado o MACBETH (Measuring Attractiveness by a Categorical Based Evaluation Tecnique), um software desenvolvido por Bana e 
Costa e Vansnick (1995). Portanto, com o uso deste software foi criado para cada ponto de vista fundamental um descritor e uma função de valor. A Quadro 6 pode ilustrar a função de valor associada ao descritor PVF8-Visão do Negócio.

Quadro 6 - Descritor e função de valor do PVF8: Visão do Negócio

\begin{tabular}{|c|l|c|c|}
\hline $\mathbf{N}$ & \multicolumn{1}{|c|}{ Des critor PVF8- Vis ão do Negócio } \\
N4 & $\begin{array}{l}\text { Os dirigente possuem argumentos convincentes que } \\
\text { justificam os inves timentos na empresa }\end{array}$ & FV \\
\hline $\mathbf{N} 3$ & $\begin{array}{l}\text { Os dirigente possuem argumentos discutíveis que } \\
\text { justificam os inves timentos na empresa }\end{array}$ & $\mathbf{B}$ & $\mathbf{1 0 0}$ \\
\hline $\mathbf{N} 2$ & $\begin{array}{l}\text { Os dirigente possuem argumentos duvidosos que } \\
\text { justificam os inves timentos na empresa }\end{array}$ & $\mathbf{N}$ & $\mathbf{0}$ \\
\hline $\mathbf{N 1}$ & $\begin{array}{l}\text { Os dirigente não possuem argumentos que } \\
\text { justificam os inves timentos na empresa }\end{array}$ & $\mathbf{- 1 8 0}$ \\
\hline
\end{tabular}

Fonte: Autoria própria (2007)

Construído os descritores e a função de valor de cada ponto de vista fundamental, foi possível avaliar localmente o desempenho de cada alternativa. Agora o avaliador pode agregar estas informações locais de modo a obter uma avaliação global. Para que fosse possível esta agregação foi preciso determinar as taxas de compensação. Taxa de compensação (vulgarmente chamada de peso) são parâmetros que os avaliadores julgaram adequados para agregar, de forma compensatória, desempenhos locais em um desempenho global (ROY,1986). Utilizando o software de Bana e Costa e Vansnick (1995) foi gerada uma escala cardinal que através de procedimentos de transformação linear foi capaz de determinar as taxas de compensação entre os pontos de vistas fundamentais. A Quadro 7 pode ilustrar o resultado final da apuração das taxas de compensação.

Quadro 7 - Taxas de compensações na estrutura de valor

\begin{tabular}{|c|c|c|c|}
\hline \multicolumn{2}{|c|}{ Avaliação qualitativa } & \multicolumn{2}{|c|}{ Taxa Comp } \\
\hline & Contexto & & $23 \%$ \\
\hline & PVF1-Produto interno bruto & $42 \%$ & \\
\hline & PVF2-Inflação & $34 \%$ & \\
\hline & PVF3-Juros reais & $24 \%$ & \\
\hline & Negócio & & $27 \%$ \\
\hline & PVF4-Setor econômico & $32 \%$ & \\
\hline & PVF5-Clientes & $28 \%$ & \\
\hline & PVF6-Concorrentes & $23 \%$ & \\
\hline & PVF7-Fornecedores & $17 \%$ & \\
\hline & Capacidade dos dirigentes & & $33 \%$ \\
\hline & PVF8-Visão de negócio & $44 \%$ & \\
\hline & PVF9-Experiência & $33 \%$ & \\
\hline & PVF10-Honestidade & $23 \%$ & \\
\hline & Riscos potenciais & & $17 \%$ \\
\hline & PVF11-Garantias & $100 \%$ & \\
\hline
\end{tabular}

Fonte: Autoria própria (2007). 
De posse das taxas de compensação utilizou-se a fórmula de agregação aditiva (KEENEY e RAIFFA, 1993). O objetivo desta fórmula é somar o desempenho da empresa em seus múltiplos critérios em um único desempenho, conforme apresenta a Equação 1:

\begin{tabular}{|l|}
\hline $\mathbf{V ( a )}=\mathbf{W 1}$. V1(a) + W2. V2 (a) + ... + Wn. Vn (a) \\
\hline $\mathrm{V}(\mathrm{a})$ = valor global da alternativa "a" \\
\hline $\mathrm{Wn}=$ taxa de compensação nos "n" PV \\
\hline Vn (a) = valor parcial da alternativa "a" nos "n" PV \\
\hline
\end{tabular}

A fórmula de agregação aditiva (Equação1) fornece a soma ponderada dos valores parciais obtidos de uma determinada alternativa em seus diversos critérios, sendo que a ponderação foi feita pelas taxas de compensação, também de cada critério. Para que se tenha uma compreensão melhor da aplicação da fórmula, utilizou-se um quadro que resume as principais informações distribuídas da seguinte maneira: PVF-ponto de vista fundamental; D-descritores; FV-função de valor e TC-taxa de compensação. A Quadro 8 ilustra o modelo de avaliação de desempenho qualitativo da Alfatecnoquímica:

Quadro 8 - Modelo de avaliação de desempenho qualitativo

\begin{tabular}{|c|c|c|c|c|c|c|c|c|c|c|c|}
\hline PVF & D & FV & TC & PVF & D & FV & TC & PVF & D & FV & TC \\
\hline Contexto & & & $\mathbf{0 , 2 3}$ & Negócio & & & 0,27 & Cap Dir & & & $\mathbf{0 , 3 3}$ \\
\hline \multirow[t]{6}{*}{ PIB } & N5 & 133 & 0,42 & Setor & N5 & 131 & 0,32 & Visão & N4 & 160 & 0,44 \\
\hline & N4 & 100 & & & N4 & 100 & & & N3 & 100 & \\
\hline & N3 & 50 & & & N3 & 69 & & & N2 & 0 & \\
\hline & N2 & 0 & & & N2 & 0 & & & N1 & -180 & \\
\hline & N1 & -67 & & & N1 & -138 & & & & & \\
\hline & & & & & & & & Exper & N4 & 175 & 0,33 \\
\hline \multirow[t]{6}{*}{ Inflação } & N5 & 138 & 0,34 & Clientes & N4 & 175 & 0,28 & & N3 & 100 & \\
\hline & N4 & 100 & & & N3 & 100 & & & N2 & 0 & \\
\hline & N3 & 50 & & & N2 & 0 & & & N1 & -200 & \\
\hline & N2 & 0 & & & N1 & -100 & & & & & \\
\hline & N1 & -100 & & & & & & Honest & N3 & 100 & 0,23 \\
\hline & & & & & & & & & $\mathrm{N} 2$ & 0 & \\
\hline \multirow[t]{11}{*}{ Jreais } & N5 & 138 & 0,24 & Concor & N5 & 157 & 0,23 & & N1 & -167 & \\
\hline & N4 & 100 & & & N4 & 100 & & & & & \\
\hline & N3 & 50 & & & N3 & 57 & & & & & \\
\hline & N2 & 0 & & & N2 & 0 & & PVF & D & FVF & TC \\
\hline & N1 & -100 & & & N1 & -114 & & Risco & & & 0,17 \\
\hline & & & & & & & & Garantia & N5 & 138 & 1 \\
\hline & & & & Fornec & N5 & 157 & 0,17 & & N4 & 100 & \\
\hline & & & & & N4 & 100 & & & N3 & 50 & \\
\hline & & & & & N3 & 57 & & & N2 & 0 & \\
\hline & & & & & N2 & 0 & & & N1 & -100 & \\
\hline & & & & & N1 & -114 & & & & & \\
\hline
\end{tabular}

Fonte: Autoria própria (2007). 
A Alfatecnoquímica foi avaliada em cada critério o que resultou nos seguintes níveis de impactos: PIB N1:-67; Inflação N4:100; Juros reais N1:-100; Setor N3:69; Clientes N3: 100; Concorrentes N2:0; Fornecedores N3:57; Visão N2:0; Experiência N3:100; Honestidade N3:100 e Garantias N2:0. Portanto, ao aplicar a fórmula de agregação aditiva a Alfatecnoquímica apresentou um valor global de referência para suas negociações de 30,4 pontos, calculado da seguinte forma:

Valor global da Alfatecnoquímica $=[0,23 \times(0,42 \times-67)+(0,34 \times 100)+(0,24 \times-100)]+$ $[0,27 \times(0,32 \times 69)+(0,28 \times 100)+(0,23 \times 0)+(0,17 \times 57)]+[0,33 \times(0,44 \times 0)+(0,33 \times 100)+$ $(0,23 \times 100)]+[0,17 \times(1 \times 0)]=30,4$ pontos.

A pontuação de 30,4 é o resultado da quantificação global de todos os pontos de vistas qualitativos e subjetivos que o avaliador julga fundamental para avaliação dos seus investimentos. Com esta referência quantificada é que será possível medir os impactos no fluxo de caixa descontado para que o avaliador ajuste ou não o valor do negócio.

Com uso de um gráfico foi possível visualizar o desempenho qualitativo da Alfatecnoquímica em cada ponto de vista fundamental, conforme apresenta a Figura 1:

Figura 1 - Perfil de impacto qualitativo da empresa em cada PVF

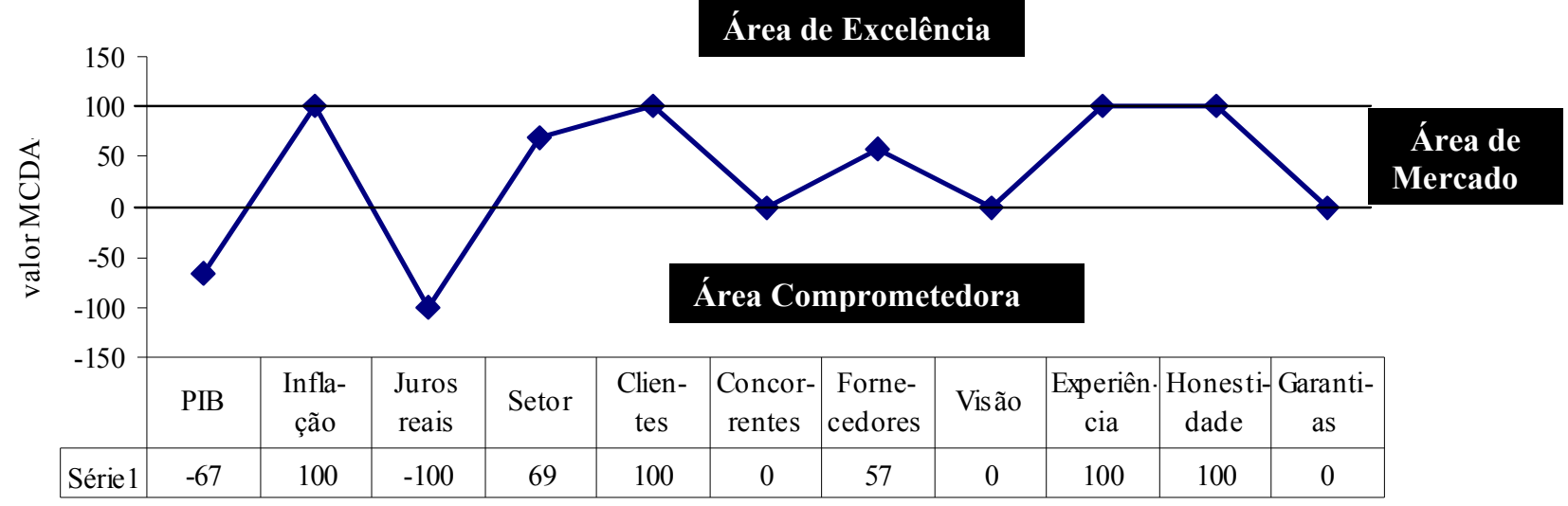

Fonte: Autoria própria (2007).

Construído o perfil de desempenho qualitativo da Alfatecnoquímica pode-se agregar estas informações no resultado final da aplicação do método do fluxo de caixa descontado, ajustando para cima ou para baixo o valor da empresa, de acordo com a escala de preferência qualitativa gerada pelo avaliador. A Quadro 9 apresenta as diversas faixas de referência: 


$\begin{aligned} & \text { Quadro 9 - Escala de preferência qualitativa } \\
& \text { Valor MCDA }\end{aligned}$
\begin{tabular}{|l|l|l|}
\hline \multicolumn{1}{|c|}{ Decis ão } \\
\hline abaixo de -150 & descartar a empresa \\
\hline de -150 a -100 & descontar no preço $80 \%$ (valor residual) \\
\hline de -100 a -50 & descontar no preço $60 \%$ \\
\hline de -50 a 0 & descontar no preço $40 \%$ & \\
\hline de 0 a 50 & considerar só o preço do FCD & \\
\hline de 50 a 100 & valorar o preço em $5 \%$ & Área de risco \\
\hline de 100 a 150 & valorar o preço em $20 \%$ & \\
\hline acima de 150 & valorar o preço em $30 \%$ & \\
\hline
\end{tabular}

Fonte: Autoria própria (2007).

No caso da Alfatecnoquímica o fluxo de caixa operacional genuíno, segundo Assaf Neto (2006), ou simplesmente fluxo de caixa da empresa, gerou o valor de $\$ 1.736 .424$, conforme Quadro 4. O seu valor global resultou em 30,4 pontos e comparado com a escala de preferência qualitativa (Quadro 9) este valor ficou entre 0 a 50 pontos (primeiro intervalo de mercado). Neste caso o avaliador permaneceria com o valor de referência somente aquele calculado pelo método do fluxo de caixa descontado, isto é, \$1.736.424, conforme apresenta a Quadro 4. Para o avaliador, os aspectos qualitativos e subjetivos inserido neste intervalo ( 0 a 50 pontos) não foram capazes de alterar o valor calculado pelo fluxo de caixa descontado.

Duas outras empresas foram testadas e incluídas na análise final dos resultados, uma do ramo farmacêutico e outra do ramo de turismo. Os resultados apurados estão representados na Quadro 10.

Quadro 10 - Contribuição da MCDA no FCD

\begin{tabular}{llclr}
\multicolumn{1}{c}{ Setores } & \multicolumn{1}{c}{ FCD } & MCDA & \multicolumn{1}{c}{ Decisão } & Valor final \\
Químico & $\$ 1.736 .424$ & 30,4 & considera o preço do FCD & $\$ 1.736 .424$ \\
Farmacêutico & $\$ 2.942 .790$ & $-45,3$ & descontar no preço 40\% & $\$ 1.765 .674$. \\
Turismo & $\$ 832.541$ & 72,1 & valorar o preço em 5\% & $\$ 874.168$ \\
\hline
\end{tabular}

Fonte: Autoria própria (2007).

No caso da Empresa Farmacêutica (A DERMATOLÓGICA - Farmácia de Manipulação) seu valor final foi ajustado com um deságio de $40 \%$. Este ajuste foi em função do uso da fórmula de agregação aditiva, cujo valor global de referência resultou em $-45,3$ pontos. Este valor comparado com a escala de preferência qualitativa (Quadro 9) ficou no primeiro intervalo de mercado (-50 a 0 pontos), considerado comprometedor. Dentro deste mesmo raciocínio, porém de forma inversa, a Empresa de Turismo (Plazatur - Agência de Turismo) teve seu valor final valorado em 5\%. Um ajuste considerado pequeno pelo avaliador, porém incentivador nas rodadas de negociação, por motivo de que os impactos ocorridos no método do fluxo de caixa descontado, através dos aspectos qualitativos e subjetivos (72,1 pontos) ficaram pouco acima do segundo intervalo de mercado (50 a 100 pontos). Isto pode ser melhor interpretado visualizando os resultados contidos na Quadro 10. 
Ao final da análise o avaliador adquiriu maior compreensão do problema e procurou aperfeiçoar os impactos das variáveis por ele consideradas fundamentais e complementares no FCD. Para tanto, transformou sua escala de preferência qualitativa (Quadro 9) em uma função de valor, representada por um polinômio de sexto grau, conforme Figura 2.

Utilizando os valores resultantes da MCDA (Quadro 10) o avaliador obteve um novo reajuste no preço final do negócio, conforme apresenta a Quadro 11. Desta forma, o avaliador consegue ajustar o valor final do negócio com qualquer valor de referência obtido no cálculo das suas preferências através da MCDA. Finalmente, o avaliador pode propor uma gama de preços ao vendedor permitindo maior flexibilidade nas rodadas de negociações.

\section{Considerações Finais}

A avaliação de empresas de grande porte, especialmente as de capital aberto, cujas informações estão prontamente disponíveis, pode ser considerada uma tarefa difícil e árdua. Em se tratando de empresas de pequeno porte, as dificuldades parecem crescer de forma exponencial.

Figura 2 - Função de valor de preferência qualitativa

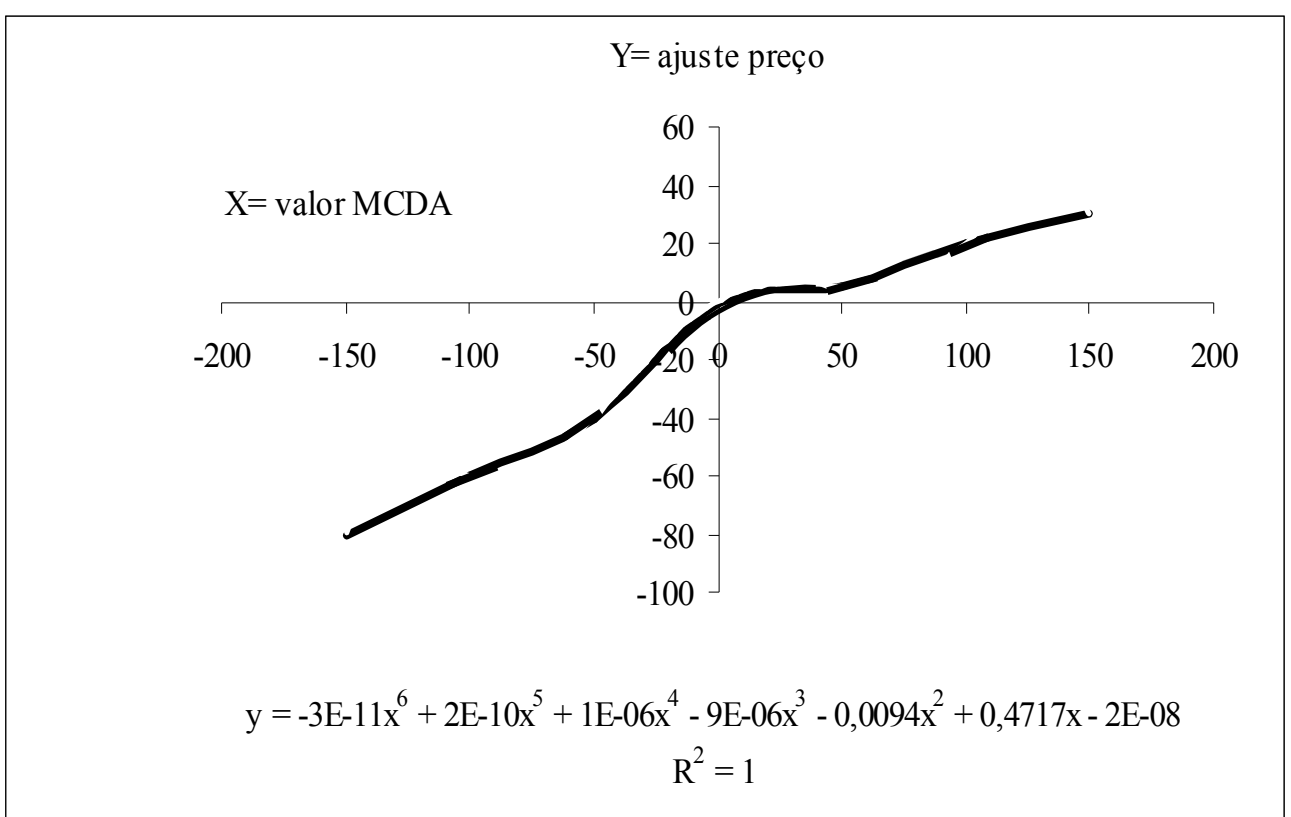

Fonte: Autoria própria (2007).

Quadro 11 - Valor final ajustado com base na função de valor

\begin{tabular}{llclr}
\multicolumn{1}{c}{ Setores } & FCD & MCDA-C & \multicolumn{1}{c}{ Decisão } & Valor final \\
Químico & $\$ 1.736 .424$ & 30,4 & valorar o preço em 6\% & $\$ 1.840 .609$ \\
Farmacêutico & $\$ 2.942 .790$ & $-45,3$ & descontar no preço 36\% & $\$ 1.883 .386$ \\
Turismo & $\$ 832.541$ & 72,1 & valorar o preço em 5\% & $\$ 874.168$ \\
\hline
\end{tabular}

Fonte: Autoria própria (2007). 
Neste sentido, parece que os métodos tradicionais de avaliação de empresas, cuja abordagem é meramente quantitativa, estão andando na contramão das características e especificidades das empresas de pequeno porte, uma vez que os aspectos qualitativos e subjetivos existem em abundância e os dados quantitativos são carentes de informações precisas e transparentes.

Embora o método do fluxo de caixa descontado seja uma forma de avaliação de empresas consistente, quando se possui valores confiáveis, há que se considerar a sua principal vulnerabilidade, ou seja, o valor de referência utilizado na avaliação de empresas tem como dimensão única a visão econômica e financeira.

Diante dos vários contextos, aqui relatados, o artigo se propôs a utilizar a MCDA cujo objetivo é fazer com que este conjunto de métodos e técnicas possam contribuir com o método do FCD na incorporação de variáveis qualitativas e subjetivas inerentes ao avaliador. Como conseqüência desta simbiose o valor de referência poderá conter uma multiplicidade de variáveis, permitindo que o avaliador elabore suas estratégias de negociação com maior competitividade.

E, para finalizar, os autores deste artigo corroboram com Martelanc, Pasin e Cavalcante (2005) em considerar que nenhum modelo fornece um valor preciso e único para uma empresa, mas sim uma estimativa de valor. Isso porque as decisões que afetam receitas, custos, despesas, capital de giro e investimentos, aliadas às mudanças ocorridas na conjuntura econômica local e global, influenciam nos resultados da empresa e alteram seu valor. Dependendo do modelo adotado, das premissas e dos cenários criados, dois avaliadores podem obter valores diferentes para a mesma empresa. Neste sentido, a avaliação de empresas não precisa ter como objetivo a fixação de um valor exato pelo qual elas podem ser negociadas, mas sim o estabelecimento de uma faixa de valores.

\begin{abstract}
In search of an assessment on business more broadly, it was necessary to incorporate the discounted cash flow variables are qualitative and subjective. Therefore, the aim was to contribute to the implementation of the method of discounted cash flow, using the methodology of multicriteria decision aid. To achieve this objective was a descriptive study of exploratory nature applied to a multicase. The intervention was in small businesses from the chemical, pharmaceutical and tourism. As a result, the appraiser set the price of the business taking into account the result of mixing between the two methodologies.
\end{abstract}

Key-words: multicriteria methodology; discounted cash flow; balance inventoried; small businesses.

\title{
Referências
}

ASSAF NETO, A. Finanças corporativas e valor. 2. ed. São Paulo: Atlas, 2006.

BANA e COSTA, C. A ; VANSNICK, J. C. Uma nova abordagem ao problema de construção de uma função de valor Cardinal: MACBETH. Investigação Operacional, v. 15, junho, 1995. 
BANCO CENTRAL DO BRASIL. Relatório de Inflação de março de 2007. Disponível em: http://www.bcb.gov.br/htms/relinf/port/2007/03/ri200703c6p.pdf. Acesso em: 01 abr. 2007.

BELTON, V. Multiple criteria decision analysis - practically the only way to choose. In: BOUYSSOU, D. Modeling inaccurate determination, uncertainty, imprecision using multiple criteria. In : LOCKETT, A. G. ; ISLEI, G. (Eds.). Improving Decision Making in Organizations, Berlin: Spriger, 1989.

CAIXA ECONÔMICA FEDERAL. Avaliação básica de crédito microempresa e pequena empresa. Material utilizado pela CEF, 2000.

COPELAN, T; KOLLER, T; MURRIN, J. Avaliação de Empresas "Valuation": calculando e gerenciando o valor das empresas. São Paulo: Makron Books, 2000.

CUPERtino, C. M; COSTA JR, N. A. C; COELhO, R. A; MENEZES, E. A. Cash Flow, Earnings and Dividends: a comparison between different valuation methods for brazilian companies. $30^{\circ}$ Encontro da ANPAD. Salvador/BA-Brasil: setembro de 2006.

DAMODARN, A. Avaliação de Investimentos: ferramentas e técnicas para a determinação do valor de qualquer ativo. Rio de Janeiro: Qualitymark, 1999.

GOODWIN, P.; WRIGHT, G. Decision Analysis for management judgment. Chichester: John Wiley e Sons, 1991. IUDÍCIBUS, S. Teoria da contabilidade. 5. ed. São Paulo: Atlas, 1997.

KASSAI, J; KASSAI, S. Balanço perguntado: solução para as pequenas empresas. Área temática: A mensuração e gestão de custos para micro, pequenas e médias empresas. XI Congresso Brasileiro de Custos. Porto Seguro, Bahia. Julho, 27 a 30, 2004.

KEENEY, R. L. Value-focused thinking: a path to creative decision-making. Cambridge: Harvard University Press, 1996.

KEENEY, R. L.; RAIFFA, H. Decision with multiple objetives, preferences, and value tradeoffs. Cambridge: University Press, 1993.

MARTLANC, R; PASIN, R; CAVALCANTE, F. Avaliação de empresas: um guia para fusões e aquisições e gestão de valor. São Paulo: Pearson Prentice Hall, 2005.

PRATT, S. Valuing a Business: The analysis and appraisal of Closely held Companies. Home wood: Irwin. 1989.

ROY, B. Multi-criteria methodology for decision aiding. Lamsade, Universitè Paris. Dauphine, cap.4, 1996.

SANTOS, J. O. Avaliação de empresas: cálculo e interpretação do valor das empresas: um guia prático. São 
Paulo: Saraiva 2005 .

SOUSA, A. F; BASTOS, D. D; MARTELANC, R. Avaliação de Empresas Brasileiras de Capital Fechado. VI SEMEAD-Seminário em Administração e Programação: ensaio em finanças. Escola de Administração (EAD), Faculdade de Economia e Administração (FEA) da Universidade de São Paulo (USP). São Paulo, março de 2003.

TOMAZONI, T; MENEZES, E. A. Estimativa do custo de capital de empresas brasileiras de capital fechado (sem comparáveis de capital aberto). Revista de Administração-RAUSP. São Paulo.

\section{Dados dos autores}

\section{Nome completo: Marcus Vinicius Andrade de Lima}

Filiação institucional: Universidade do Sul de Santa Catarina - Unisul

Departamento: Mestrado em Administração da Unisul

Função ou cargo ocupado: Professor Pesquisador

Endereço completo para correspondência: Rua Crispim Mira, 97, ap. 701. Centro. CEP: 88020-540. Florianópolis, Santa Catarina. Brasil.

Telefones para contato: (48) 99718979 / (48) 32291932 / (48) 32221590

e-mail:marcus.lima@unisul.br

\section{Nome completo: Ana Lúcia Miranda Lopes}

Filiação institucional: Universidade Federal de Minas Gerais - UFMG

Departamento: Ciências Administrativas - CAD

Função ou cargo ocupado: Professora Adjunta - Pesquisadora

Endereço completo para correspondência (bairro, cidade, estado, país e CEP): Avenida Antônio Carlos 6627

- FACE - Sala 4042 Pampulha - CEP 31270-901- Belo Horizonte - MG. Brasil.

Telefones para contato: (31) 34097317 / Fax (31) 34097039

e-mail: analopes@,face.ufmg.br

\section{Nome completo: Ademar Dutra}

Filiação institucional: Universidade do Sul de Santa Catarina - Unisul

Departamento: Mestrado em Administração da Unisul

Função ou cargo ocupado: Professor Pesquisador

Endereço completo para correspondência: Rua Trajano, 219. Centro. CEP: 88010 - 010 - Florianópolis, SC, Brasil.

Telefones para contato: (48) 32291932

e-mail: marcus.lima@unisul.br 